\title{
Non-invasive Thoracic Impedance Changes in COVID-19 Pulmonary Infection
}

\author{
Sunil Kapur ${ }^{1} \cdot$ Michael O. Sweeney $^{1} \cdot$ William Sauer $^{1} \cdot$ Calum A. MacRae $^{1}$ \\ Received: 29 May 2020 / Accepted: 1 October 2020 / Published online: 13 October 2020 \\ (C) Springer Science+Business Media, LLC, part of Springer Nature 2020
}

Keywords Thoracic impedance $\cdot$ Implantable cardiac defibrillator $\cdot$ Covid-19

$\begin{array}{ll}\text { Abbreviations } \\ \text { TI } & \text { Thoracic impedance } \\ \text { ICD } & \text { Implantable cardiac defibrillator } \\ \text { COVID-19- } & \text { Coronavirus disease } 19 \\ \text { HF } & \text { Heart failure } \\ \text { EC } & \text { Electrical cardiometry } \\ \text { EIT } & \text { Electrical impedance tomography }\end{array}$

Severe acute respiratory syndrome coronavirus 2 (SARSCoV-2) causes coronavirus disease 2019 (COVID-19), a systemic illness with respiratory tract abnormalities [1]. Thoracic impedance (TI) is a capability of implantable cardiac defibrillators (ICDs) that reflects complex biophysical characteristics in the chest. Dynamic changes in TI have been shown to reflect changes in pulmonary interstitial fluid in heart failure (HF) patients $[2,3]$. We sought to determine if COVID-19related pulmonary changes could result in changes in TI as measured by ICDs. Consecutive patients followed by the Brigham and Women's Cardiac Electrophysiology Device Clinic who were evaluated for COVID between March 15 and April 15, 2020, were identified and divided into two cohorts: those with severe pulmonary disease requiring hospitalization and those without who were managed out of hospital. Those diagnosed with COVID-19 via PCR testing and an implanted device with TI monitoring capability were selected. Patients were excluded from this study if they had evidence of decompensated heart failure, recent device implant, advanced renal failure \pm dialysis (chronic kidney disease class 4 or 5

Editor-in-Chief Enrique Lara-Pezzi oversaw the review of this article

Sunil Kapur

skapur@bwh.harvard.edu

1 Cardiovascular Division, Brigham and Women's Hospital, 75 Francis Street, Boston, MA 02115, USA with an estimated glomerular filtration rate (GFR) of $<30 \mathrm{~mL} /$ min), or pulmonary embolism. With these criteria, the study consisted of 3 hospitalized and 8 nonhospitalized patients. Study approval was obtained from the Mass General Brigham Institutional Review Board.

Among the hospitalized cohort, patient 1 is a 73-year-old gentleman with ischemic cardiomyopathy (LVEF 30\%). He was admitted on February 2, 2020, for heart failure exacerbation. The patient was optimized with diuresis/medication during which a reduction of central venous pressure from 31 of $12 \mathrm{mmHg}$. NT-proBNP (reference $<1800 \mathrm{pg} / \mathrm{mL}$ ) decreased from $20,609 \mathrm{pg} / \mathrm{mL}$ to baseline of $4124 \mathrm{pg} / \mathrm{mL}$. The patient had improvement and was discharged on March 3 . The patient reported fevers and chills starting March 5 and shortness of breath on March 8. He presented to the hospital on March 10 and was hypoxic at $92 \%$ saturation on room air. COVID testing was positive on March 11 and again on March 12. On March 16, he developed worsening hypoxia and was intubated. Chest CT showed parenchymal lung disease consistent with COVID. He recovered with intensive level care over the course of the 7 days and was extubated March 23 . Throughout his course, there was no clinical indication of recurrent heart failure, and the patient had continued medication adherence. His CVP was measured via central line during his intensive care course (March 16-27) with readings 10$14 \mathrm{mmHg}$ by manometry. NT-proBNP remained at his optimized baseline (3782 on March 11 and 4252 on March 24). Repeat COVID-19 testing on April 5 was negative. He continued to exhibit an oxygen requirement and was discharged to a rehabilitation facility on $4 \mathrm{~L}$ nasal cannula which was ultimately discontinued on April 8, 2020. He was discharged home feeling well on April 12, 2020. A routine-scheduled remote transmission on April 15 was performed. TI had decreased from $66 \Omega$ to a nadir of $49 \Omega$ during the time course as noted in Fig. 1a. Reference impedance, calculated by the device as a moving average, is also displayed. 
a

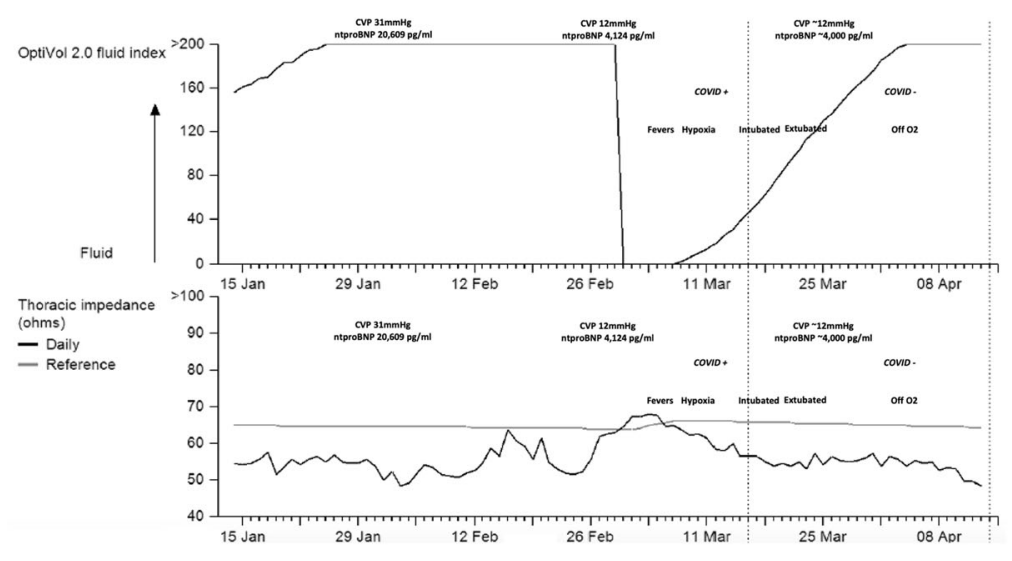

b Normalized Thoracic I Impedance over Time (days)

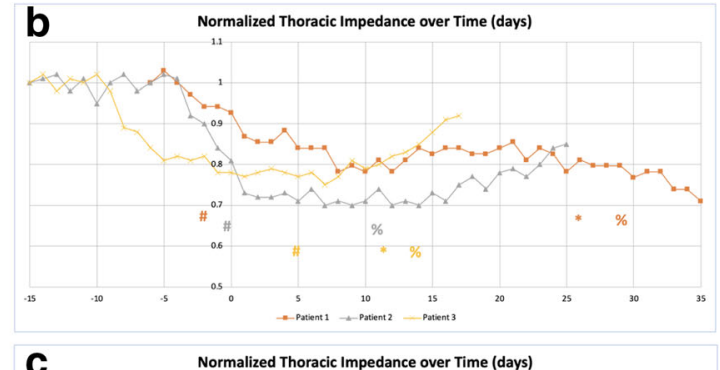

C

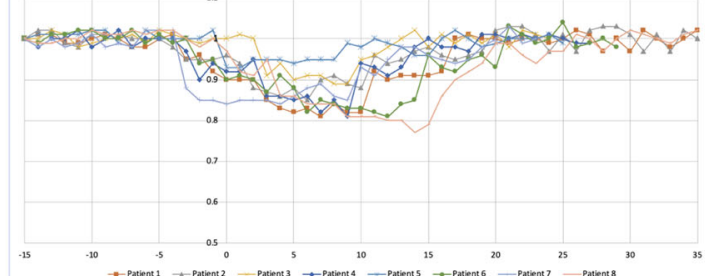

Fig. 1 a Example of thoracic impedance of a patient with COVID pulmonary infection requiring intubation. Top panel is Optivol 2.0 (proprietary Medtronic Inc. thoracic impedance alert). Bottom panel is raw thoracic impedance $(\Omega)$. Annotation of clinical events including preceding heart failure hospitalization followed by COVID-19 infection. b Normalized thoracic impedance $(\Omega / \Omega)$ of patients 1,2 , and 3 with known COVID-19 infection requiring inpatient hospitalization over time (days). Day $=0$ first positive COVID test. Thoracic impedance is normalized to a steady baseline from day -45 to -15 in patients 2 and 3 and from a baseline -8 to -6 in patient 1 (see text). \# = onset of respiratory symptoms; $\%=$ resolution of respiratory symptoms. $*=$ first negative COVID test. $\mathbf{c}$ Normalized thoracic impedance $(\Omega / \Omega)$ of patients 1-8 with known COVID-19 infection not requiring hospitalization/ supplemental oxygen over time (days). Day $=0$ first positive COVID test. Thoracic impedance is normalized to a steady baseline from day -45 to -15 days
Patients 2 is a 66-year-old woman with hypertrophic cardiomyopathy status post ICD in 2018 for primary prevention with PCR-positive infection requiring intubation. Patient 3 is a 34-year-old gentleman with long QT syndrome status post ICD in 2013 for secondary prevention with PCR-positive infection requiring intubation. Chest CTs showed parenchymal lung disease consistent with COVID. Figure $1 \mathrm{~b}$ shows TI for patients 1-3 normalized to day 0 being first positive COVID19 test with an average prior impedance for 45 to 15 days prior to day 0 being an impedance of 1.00. In hospitalized patients, the time prior to diagnosis that the TI started to decrease ranged from 3 to 8 days prior to PCR diagnosis.

Eight additional patients were identified with COVID-19 who did not require hospitalization nor require supplemental oxygen. Figure $1 \mathrm{c}$ shows raw TI normalized in time and impedance. The nadir-normalized TI for nonhospitalized patients was $0.84 \pm 0.05$, compared with the average lowest normalized TI for hospitalized patients which as noted was 0.72 \pm 0.02 . The time from diagnosis to the nadir was $8 \pm 4$ days in nonhospitalized patients and $17 \pm 15$ days in hospitalized patients. The time from diagnosis to normalization of TI (defined as 2 days of normalized $\mathrm{TI}>0.95$ ) was $13 \pm 5$ days in nonhospitalized patients, whereas none of the three hospitalized patients had normalized at the end of follow-up (ranging from 17 to 35 days of follow-up).

Impedance is a measure of the degree a substance that resists the flow of electrical current. In implanted defibrillators, the "circuit" traverses from the lead in the right ventricular apex to the device itself (often in the left pre-pectoral area) which therefore incorporates significant amount of lung tissue. Compared with the high resistivity of thoracic tissue ( $\rho=$ 200-5000 $\Omega$ ), blood and fluid ( $\rho=65-150 \Omega$ ) provide much lower resistance to current. Thus, if the circuit accumulates higher fluid content, as in heart failure, a lower impedance will be noted. This physical basis has been exploited to assess hemodynamic measurements and changes in fluid accumulation in the setting of congestion. Changes in TI are more important than a baseline, and therefore daily changes are remotely monitored in patients predisposed to heart failure. While designed for monitoring interstitial fluid content of the lung parenchyma in heart failure, the concept of TI might extend to other pulmonary processes. A 2010 study reported that changes in TI during continuous measurement with implantable devices can detect evolving bacterial pneumonia [4]. Pathologic examinations taken from COVID patients early in the disease (prior to symptoms) revealed edema, proteinaceous exudate, focal reactive hyperplasia of pneumocytes with patchy inflammatory cellular infiltration, and multinucleated giant cells [5]. Some or all of these findings might explain TI changes observed prior to the COVID-19 diagnosis. The degree of reduction is greater, and its duration is longer in hospitalized patients. It is possible that the extent of TI reduction may offer a surrogate of disease severity. Importantly, in our small cohort for severe forms of COVID-19, the decrease in TI precedes diagnosis by 3- 8 days, and this could be an early warning sign. Our results should be interpreted with caution, since they refer to a small population. These findings suggest that TI might be utilized for COVID-19 management. 


\section{Compliance with Ethical Standards}

Conflict of Interest Dr. Kapur receives consulting fees/honoraria from Abbot Inc., Medtronic Inc., Novartis Pharmaceuticals Inc. Dr. Sweeney has received research support from, is a paid consultant for, has served on the speakers' bureau of, and has received honoraria from Medtronic, Inc. Dr. Sauer has received consulting fees and educational and research funding from Medtronic, Boston Scientific, Abbott, and Biotronik, all manufacturers of implantable cardiac defibrillators. Dr. MacRae receives consulting fees/honoraria from Astra Zeneca, Bristol Myers Squibb, and Pfizer for work in digital health and is a founder of Atman Health.

Ethical Approval All procedures performed in studies involving human participants were in accordance with the ethical standards of the institutional and/or national research committee and with the 1964 Helsinki declaration and its later amendments or comparable ethical standards.

Informed Consent Informed consent was obtained from all individual participants included in the study.

\section{References}

1. Kong, W., Li, Y., Peng, M. et al. (2020). SARS-CoV-2 detection in patients with influenza-like illness. Nature Microbiology, 5, 675678. https://doi.org/10.1038/s41564-020-0713-1.

2. Luepker, R. V., Michael, J. R., \& Warbasse, J. R. (1973). Transthoracic electrical impedance; quantitative evaluation of a non-invasive measure of thoracic fluid volume. American Heart Journal, 85(1), 83-93.

3. Wang, L. (2007). Fundamentals of intrathoracic impedance monitoring in heart failure. The American Journal of Cardiology, 99(10A), 3G-10G.

4. Barth, C., et al. (2010). Intrathoracic impedance monitoring detecting pneumonia. Clinical Research in Cardiology, 99(5), 333-335.

5. Tian, S., Hu, W., Niu, L., Liu, H., Xu, H., Xiao, S. Y. (2020). Pulmonary pathology of early-phase 2019 novel coronavirus (COVID-19) pneumonia in two patients with lung cancer. Journal of thoracic oncology: official publication of the International Association for the Study of Lung Cancer, 15(5), 700-704. https:// doi.org/10.1016/j.jtho.2020.02.010

Publisher's Note Springer Nature remains neutral with regard to jurisdictional claims in published maps and institutional affiliations. 